\title{
UWB Miniature Antenna Based on the CRLH-TL with Increasing the Gain for Advanced Electromagnetic Requirements
}

\author{
Mohammad Alibakhshi-kenari ${ }^{1}$ and Mohammad Naser-Moghadasi ${ }^{2}$ \\ ${ }^{1}$ School of Electrical and Communication Engineering, Shahid Bahonar University, Kerman, Iran \\ ${ }^{2}$ School of Electrical and Communication Engineering, Islamic Azad University, Science \& Research Branch, Tehran, Iran \\ Corresponding Author, E-mail: makenari@mtu.edu
}

\begin{abstract}
In this paper, a novel ultra-wideband (UWB) miniature antenna based on the composite right-left handed transmission line (CRLH-TL) structure with enhancing the gain is proposed and investigated. By the embedded CRLH metamaterial (MTM) technology the antenna is presented with best in bandwidth, size, efficiency and radiation patterns. To realize the antenna characteristics, the $\Pi$-shaped gaps on the rectangular radiation patches are printed by standards manufacturing techniques. This antenna is constructed of two unit cells so that covers the impedance bandwidth $\left(\mathrm{S}_{11}<\right.$ $10 \mathrm{~dB}$ ) of $2.25-4.7 \mathrm{GHz}$, which corresponding to $70.5 \%$ feasible bandwidth. The presented antenna overall size is $10.8 \mathrm{~mm} \times 6.9 \mathrm{~mm} \times 0.8 \mathrm{~mm}$ or $0.09 \lambda_{0} \times 0.05 \lambda_{0} \times 0.006 \lambda_{0}$ at the operating frequency of $\mathrm{f}=2.5 \mathrm{GHz}$ (where $\lambda_{0}$ is free space wavelength). The radiation peak gain and the maximum efficiency happened at $4.6 \mathrm{GHz}$, are $3.96 \mathrm{dBi}$ and $63.6 \%$, respectively.
\end{abstract}

\section{Introduction}

In Recent years, with development of the broadband and minimizing technology for high resolution and high data transmission rates and foot print area reduction in modern communication systems, there are increasing the demands for small low-cost antenna with unidirectional radiation patterns, dispersive and broad band characteristics. The printed antennas have received great attention in broadband applications due to their advantages of compact, planar, low cost, light weight, broadband, compatibility and easy of integration with other microstrip circuits. Applications in present-day mobile communication systems usually require smaller antenna size in order to meet the miniaturization requirements of mobile units. Thus, size reduction and bandwidth enhancement are becoming major design considerations for practical applications of microstrip antennas [1].

Metamaterial (MTM) [2], have recently been extensively discussed and studied for special properties. Metamaterials (MTMs) are manmade composite materials, engineered to produce the desired electromagnetic propagation behavior not found in natural media [2], [3]. Those unusual properties were used to improving the antennas and circuits performances. Microstrip antennas have been developed for applications in present communication systems [4], [5], but there is a fact that the size reduction levels remain unsatisfactory to the electromagnetic community. Several techniques were suggested to reduce the antenna size [6], however, such techniques usually suffer from increasing the design complexity. The occurrence of metamaterial may be a solution for this challenge [7], [8]. In this work, we have used of the metamterial technology and the simple techniques for foot print area reduction, bandwidth enhancement and improving the gain of the antenna, which consist of employing the printed planar mushroom structure based on CRLH-TL and the suitable structural parameters. Various implementations of metamaterial structures have been reported and demonstrated [2]. In this paper a metamaterial CRLH antenna including two unit cells which of each unit cells embrace of two printed $\Pi$-shaped gaps capacitors and the spiral inductor accompanying a metallic via connected to ground plane is presented. The printed $\Pi$-shaped structure exhibits the wide bandwidth, small size and good radiation properties, so that can be used as a UWB and miniaturized antenna.

This communication is organized in the follows. A UWB miniature antenna prototype with high gain and efficiency, which is implemented by new concepts are depicted in Section 2. Followed by section 3 where various performances including dimension, impedance bandwidth and radiation patterns characteristics of the antenna are demonstrated. Further discussion and conclusion are raised at last.

\section{Theory of the Proposed Antenna}

As discussed in [2], [9], several implementations can be used to realize the CRLH-TL unit cell including surface mount technology (SMT) chip components and distributed lines. However, lumped elements are not appropriate in antenna design because of their lossy characteristics and discrete values. We have used the printed planar technique for designing the desired antenna, since the printed planar 
structures due on their advantages such as foot print area reduction, loss less and non-discrete values [13-14-15] are good candidates for the antenna design. The offered UWB miniature antenna with high gain and efficiency that is based on the CRLH-TL consists of two unit cells while each of unit cells are composed of the rectangular radiation patches with two printed $\Pi$-shaped gaps on the patches, and a spiral inductor accompanying a metallic via hole connected to the ground plane. Figure 1 shows the proposed antenna geometry and Figure 2 displays the equivalent circuit model of each CRLH unit cells. As is clear, in this structure port 1 is excited with input signal and port 2 is matched to $20 \Omega$ load impedance. The antenna structure is based on a composite right-left handed (CRLH) transmission line (TL) model used as a periodic structure. Because the lowest mode of operation is a LH mode, the propagation constant approaches negative infinity at the cutoff frequency, and reduce its magnitude as frequency is increased. Using this phenomenon, an electrically large but physically small antenna is developed.

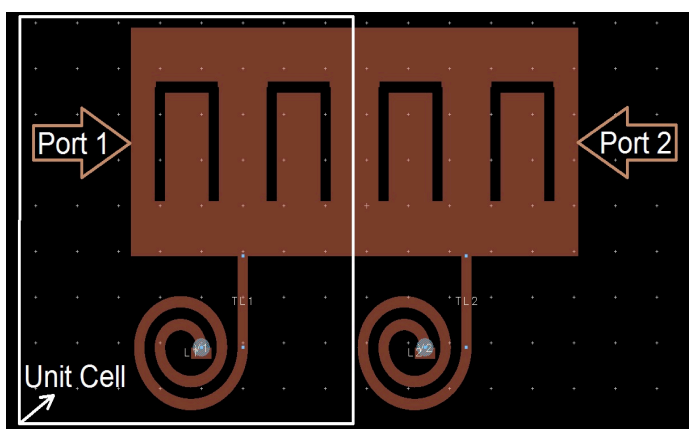

(a)

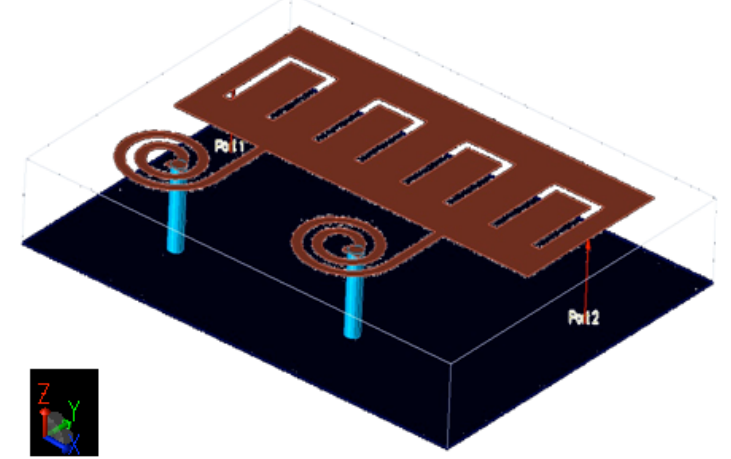

(b)

Figure.1. Configuration of the presented UWB miniature antenna combined of two unit cells based on CRLH MTMTL. a) Top view, b) Isometric view.

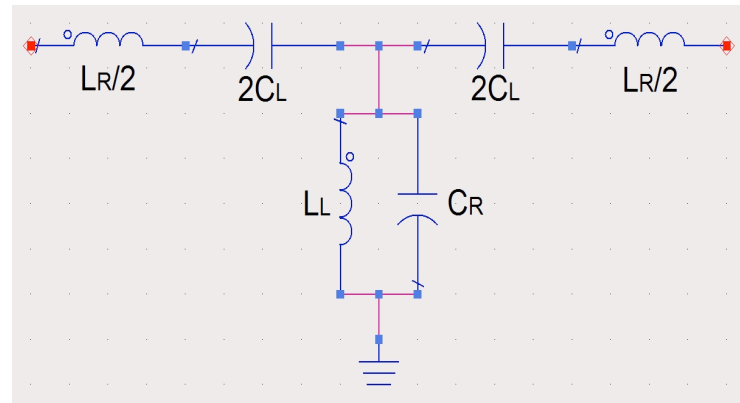

Figure.2. Equivalent circuit model of the CRLH MTM unit cell.

By implementing the $\Pi$-shaped gaps and the spiral inductors with shorting via-hole connected to ground plane, the series capacitance $\left(\mathrm{C}_{\mathrm{L}}\right)$ and shunt inductance $\left(\mathrm{L}_{\mathrm{L}}\right)$ are easily implemented in a compact fashion. The host TL possesses the right-handed parasitic effects that can be seen as the shunt capacitance $\left(C_{R}\right)$ and the series inductance $\left(L_{R}\right)$.

In this paper, we have employed of the metamaterial (MTM) technology and the printed planar approach, as a result the foot print area reduction of the proposed antenna is achieved. Overall size of this antenna is $0.09 \lambda_{0} \times 0.05 \lambda_{0} \times 0.006 \lambda_{0}$ where $\lambda_{0}$ is the free space wavelength at the operating frequency of $\mathrm{f}=2.5 \mathrm{GHz}$. By adjusting the smaller distance between edges of the printed $\Pi$-shaped gaps the wide bandwidth from $2.25 \mathrm{GHz}$ to $4.7 \mathrm{GHz}$, which corresponds to $2.45 \mathrm{GHz}$ achievable bandwidth, has been obtained. The reflection coefficient $\left(\mathrm{S}_{11}<-10 \mathrm{~dB}\right)$ is plotted in Figure 3. Furthermore, with selecting the proper number of unit cells $(N)$ and the structural parameters i.e. the spiral inductors, their number of turns $(N)$, inner radius measured to the center of the conductor $\left(R_{i}\right)$, conductor width $(W)$ and conductor spacing $(S)$ the good radiation performance have been achieved. The gain and efficiency of the proposed antenna are variable from $0.4 \mathrm{dBi}$ to $3.96 \mathrm{dBi}$ and from $19.5 \%$ to $63.6 \%$, respectively, which shown very good radiation characteristics. According to the results, the proposed antenna based on CRLH-TL made very small size and wide bandwidth to support today's multi-band modern wireless applications, mobile handsets and advanced electromagnetic requirements.

Figure 1 shows configuration of the recommended antenna constructed of two unit cells based on CRLH-TL, which was designed on a FR_4 substrate with dielectric constant of 4.6, thickness of $0.8 \mathrm{~mm}$ and Tan $\delta=0.001$. Its unit cell dimension is $5.4 \mathrm{~mm} \times 6.9 \mathrm{~mm}$ or $0.045 \lambda_{0} \times 0.05 \lambda_{0}$. In each of unit cells, the series capacitance $\left(\mathrm{C}_{\mathrm{L}}\right)$ is established by two $\Pi$-shaped gaps printed on top of the substrate by standards manufacturing techniques, and the shunt inductance $\left(\mathrm{L}_{\mathrm{L}}\right)$ arise from the spiral inductor shorted to the ground plane through the metallic via. The structure possesses the right-handed parasitic effects that can be seen as shunt capacitance $\left(C_{R}\right)$ and series inductance $\left(L_{R}\right)$. The shunt capacitance $C_{R}$ is mostly come from the gap capacitance between the patch and 
the ground plane, and the series inductance $L_{R}$ is created by unavoidable currents that flowing on the patches, which indicates that these capacitance and inductance cannot be ignored. In this structure, port 1 is excited by input signal and port 2 is matched to $20 \Omega$ load impedance, as is illustrated in Figure 1. The proposed design keeps the overall size of the unit cell compact while aims at reducing the ohmic loss to improve the gain and radiation efficiency. This antenna can support all cellular frequency bands from $2.25 \mathrm{GHz}$ to 4.7 $\mathrm{GHz}$, using single or multiple feed designs, which eliminates the need for antenna switches. These attributes make the proposed antenna is well suitable for electromagnetic requirements such as millimeter waves and emerging wireless applications [10], [11] and mobile handsets.

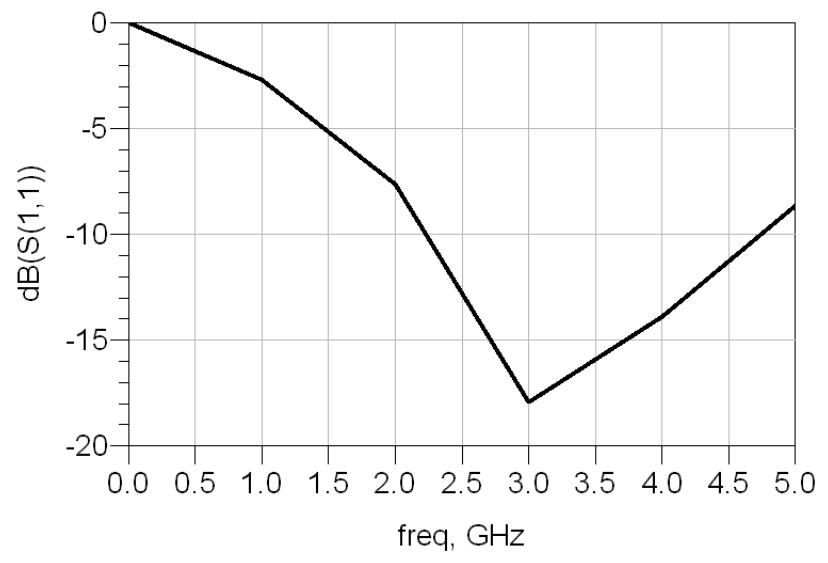

Figure.3. Reflection coefficient, $\mathrm{S}_{11}<-10 \mathrm{~dB}$.

\section{Simulation Results and Discussions}

The proposed metamaterial antenna is designed as a CRLH antenna where the substrate has dielectric constant of $\varepsilon_{r}=$ 4.6, thickness of $\mathrm{h}=0.8 \mathrm{~mm}$ and Tan $\delta=0.001$. The UWB miniature antenna is simulated by using the full-wave Agilent Advanced Design (ADS) simulator. The simulated reflection coefficient $\left(\mathrm{S}_{11}\right.$ parameter) is displayed in Figure 3 and the simulated two and three dimensional (2-D and 3-D) radiation gain patterns at 2.3, 3.4 and 4.6 GHz are plotted in Figure 4. With attention to this figure, the radiation patterns have unidirectional characteristics. The gains and radiation efficiencies at $2.3,3.4,4.6 \mathrm{GHz}$ are 0.4 and $19.5 \%, 2.8$ and $47.8 \%$, and $3.96 \mathrm{dBi}$ and $63.6 \%$, respectively. To validate the design procedure, the proposed antenna was compared with some of the conventional antennas and their dimensions and radiation characteristics are summarized in table 1 .

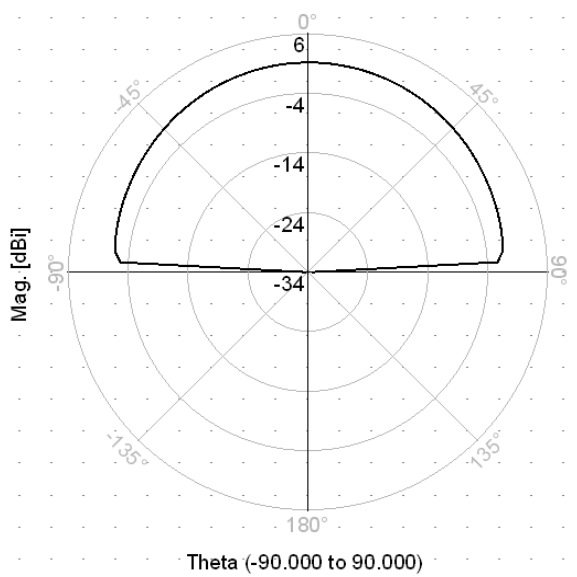

$2-\mathrm{D}$ at $2.3 \mathrm{GHz}$

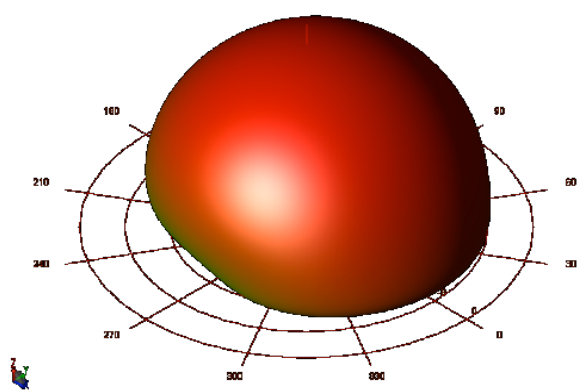

$3-\mathrm{D}$ at $2.3 \mathrm{GHz}$

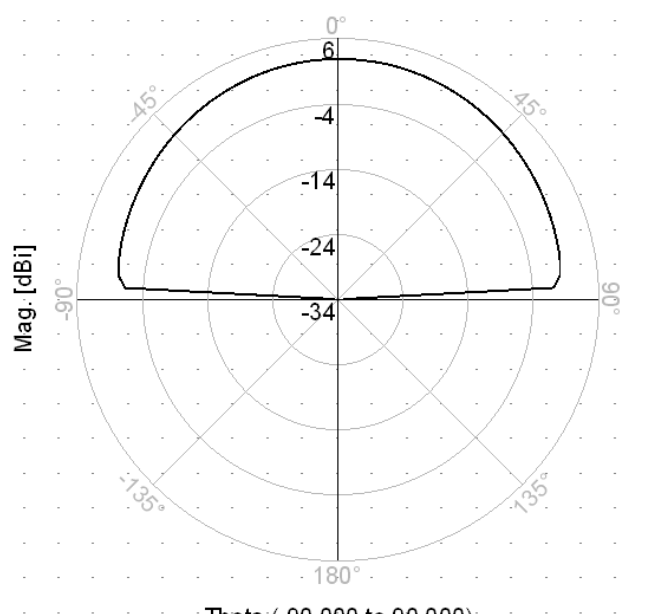

Theta $(-90.000$ to 90.000$)$.

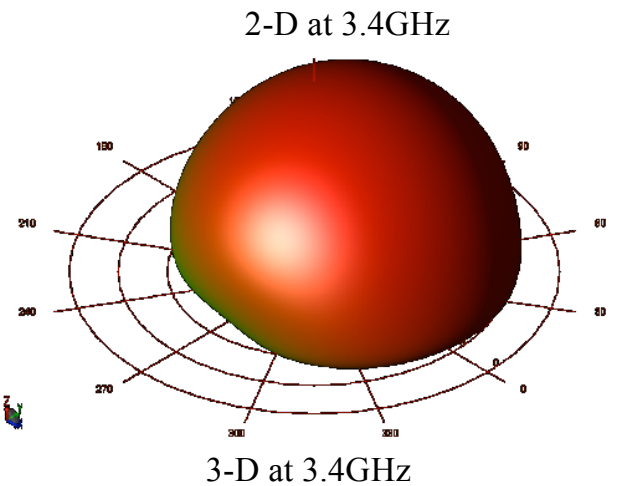



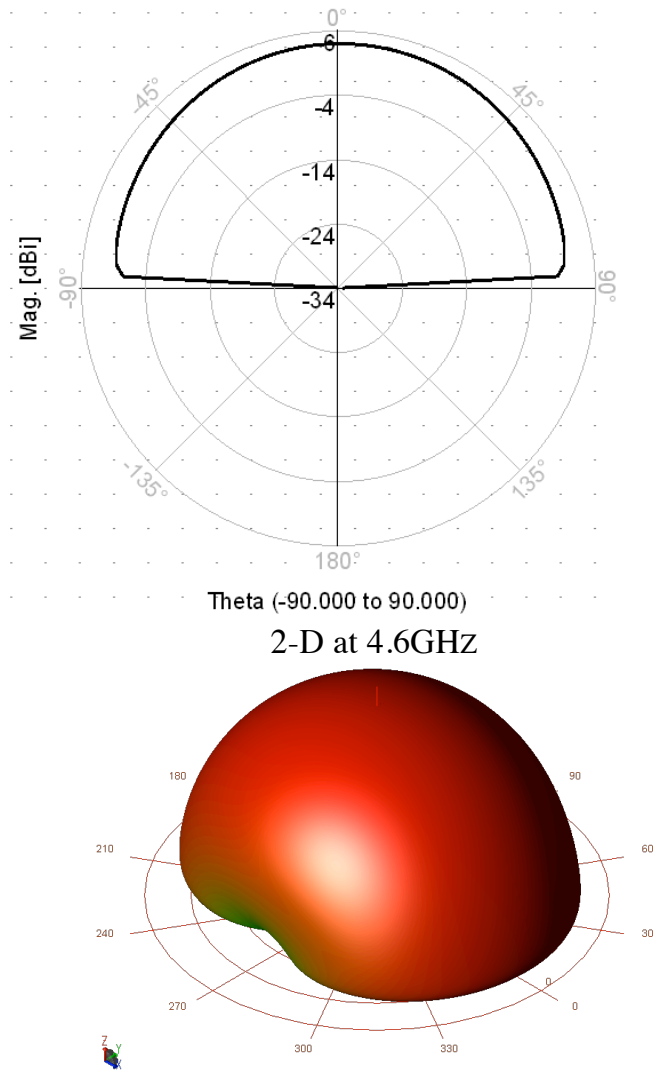

$3-\mathrm{D}$ at $4.6 \mathrm{GHz}$

Figure.4. Antenna radiation gains patterns in elevation ( $\Phi=0$ degree).

Obviously, the unit cells of the proposed antenna are designed for exhibit the frequency bandwidth from $2.25 \mathrm{GHz}$ to $4.7 \mathrm{GHz}$, while the antenna has small dimension of $10.8 \mathrm{~mm}$ by $6.9 \mathrm{~mm}$ by $0.8 \mathrm{~mm}\left(0.09 \lambda_{0} \times 0.05 \lambda_{0} \times 0.006 \lambda_{0}\right)$ along with the good matching for $20 \Omega$ impedance port. The gain and radiation efficiency of the antenna are varied from $0.4 \mathrm{dBi}$ to $3.96 \mathrm{dBi}$ and from $19.5 \%$ to $63.6 \%$, respectively.

Table 1. Dimensions and radiation characteristics of the some of the conventional antennas in comparison to the proposed antenna.

\begin{tabular}{|c|c|c|c|}
\hline Parameters & {$[11]$} & {$[12]$} & Proposed Antenna \\
\hline Gain & $0.45 \mathrm{dBi}$ & $0.6 \mathrm{dBi}$ & $3.96 \mathrm{dBi}$ \\
\hline Bandwidth & $0.8-2.5 \mathrm{GHz}$ & $1-2 \mathrm{GHz}$ & $2.25-4.7 \mathrm{GHz}$ \\
\hline Efficiency & $53.6 \%$ & $26 \%$ & $63.6 \%$ \\
\hline Dimension & $0.4 \lambda_{\infty} \times 0.03 \lambda_{\circ} \times 0.03 \lambda_{0}$ & $0.07 \lambda_{\circ} \times 0.07 \lambda_{\circ} \times 0.03 \lambda_{\circ}$ & $0.09 \lambda_{\circ} \times 0.05 \lambda_{\circ} \times 0.006 \lambda_{\diamond}$ \\
\hline
\end{tabular}

\section{Conclusions}

In this paper, we have introduced the new concepts for antenna size reduction, increase its bandwidth accompanying good radiation properties based on the metamaterial methodology. A practical UWB, miniature, high gain and radiation efficiency antenna with a simple feed structure and planar circuit integration possibilities has been demonstrated. The antenna dimension is $10.8 \mathrm{~mm} \times 6.9 \mathrm{~mm} \times 0.8 \mathrm{~mm}$ or $0.09 \lambda_{0} \times 0.05 \lambda_{0} \times 0.006 \lambda_{0}$ where $\lambda_{0}$ is free space wavelength at the operating frequency of $2.5 \mathrm{GHz}$. The reflection coefficient below $-10 \mathrm{~dB}$ from $2.25 \mathrm{GHz}$ to $4.7 \mathrm{GHz}$ was obtained, which related to $70.5 \%$ practical bandwidth. The peak gain and the maximum efficiency of the proposed antenna happened at $4.6 \mathrm{GHz}$ are $3.96 \mathrm{dBi}$ and $63.6 \%$, respectively. This antenna has the advantages of ultra-wideband, compact size, high gain and efficiency, low profile, unidirectional radiation patterns, low cost, light weight and simple of implementation. According to the obtained results, the recommended antenna can be used for the millimeter wave applications, mobile handsets, wireless communications and electromagnetic requirements.

\section{Acknowledgements}

The authors would like to express their sincere thanks to Iran Telecommunication Research Center (ITRC) with Contract number of $6987 / 500 / \mathrm{T}$, the microwave and millimeter wave laboratory of the Amirkabir University of Technology (Tehran Polytechnic), Tehran, Iran and the antenna laboratory of the K. N. Toosi University of Technology, Tehran, Iran.

\section{References}

[1] C. J. Lee, K. M. H. Leong, and T. Itoh, "Broadband Small Antenna for Portable Wireless Application," in proc. Interational Workshop on Antenna Technology: Small Antennas and Novel Metamaterials, pp. 10- 13, 2008.

[2] C. Caloz and T. Itoh, Electromagnetic Metamaterials: Transmission Line Theory and Microwave Applications, The Engineering Approach, New York, John Wiley \& Sons, 2005.

[3] R. A. Shelby, D. R. Smith, and S. Schultz, "Experimental Verifcation of a Negative Index of Refraction," Science,292, 55 14, 2001, pp. 77-79.

[4] W. L. Stutzman, Antenna Theory and Design, Second Edition: J. Wiley \& Sons, 1997.

[5] Aparna Sankarasubramaniam, "Design guidelines for tunable coplanar and microstrip patch antennas," Microwave Conference, European, 2007.

[6] K. L. Wong, Planar Antennas for Wireless Communications, Wiley-Interscience, 2003. 
[7] Andrea Alù, "Subwavelength, compact, resonant patch antennas loaded with metamaterials," IEEE Transaction on Antennas and Propagation, vol. 55, no. 1, 2007.

[8] Ourir, A, "Phase-varying metamaterial for compact steerable directive antenna," Electronics Letters, vol.43, pp. 493-494, 2007.

[9] A. Lai, C. Caloz, and T. Itoh, "Composite right/lefthanded transmission line metamaterials," IEEE Microwave., Mag., vol. 5, no. 3, pp. 34-50, sept. 2004.

[10] C. J. Lee, M. Achour, and A. Gummalla, "Compact Metamaterial High Isolation MIMO Antenna Subsystem," in proc. Asia Pacifc Microwave Conference, pp. 1-4, 2008.

[11]Y. Li, Z. Zhang, J. Zheng and Z. Feng, "Compact heptaband reconfigurable loop antenna for mobile handset," IEEE Antennas and Wireless Propagation Letters, vol. 10, pp. 1162- 1165, 2011.

[12] C. J. Lee, K. M. K. H. Leong, and T. Itoh, "Composite right/left-handed transmission line based compact resonant antennas for RF module integration," IEEE Trans. Antennas and Propagation., vol. 54, no. 8, pp. 2283-2291, Aug. 2006.

[13] Mohammad Alibakhshi-Kenari, Masoud Movahhedi and Hadi Naderian, "A New Miniature Ultra Wide Band Planar Microstrip Antenna Based on the Metamaterial Transmission Line" 2012 IEEE AsiaPacific Conference on Applied Electromagnetics (APACE 2012), December 11-13, 2012, Melaka, Malaysia.

[14] Mohammad Alibakhshi-Kenari, Masoud Movahhedi and Ahmad Hakimi, "Compact and Ultra Wide Band Planar Antenna Based on the Composite Right/LeftHanded Transmission Line Accompanying Improvement" First Iranian Conference on Electromagnetic Engineering (ICEME 2012), Tehran, Iran, December 2012.

[15] Mohammad Alibakhshi Kenari, "New TravelingWave Antenna Resonating at $6 \mathrm{GHz}$ Based on Artificial Transmission Line Metamaterial Structures for RF Portable Devices," Open Journal of Antennas and Propagation, 2013, 1, 5-10 http://dx.doi.org/10.4236/ojapr.2013.12003

Published Online September 2013 (http://www.scirp.org/journal/ojapr). 